Vol. 47 (1993) [297-306]

\title{
BICONCAVE-FUNCTION CHARACTERISATIONS OF UMD AND HILBERT SPACES
}

JINSIK MOK LEE

Suppose that $\mathbf{X}$ is a real or complex Banach space with norm $|\cdot|$. Then $\mathbf{X}$ is a Hilbert space if and only if

$$
E|x+Y| \leqslant 2
$$

for all $x$ in $\mathrm{X}$ and all $\mathrm{X}$-valued Bochner integrable functions $Y$ on the Lebesgue unit interval satisfying $E Y=0$ and $|x-Y| \leqslant 2$ almost everywhere. This leads to the following biconcave-function characterisation: $A$ Banach space $X$ is a Hilbert space if and only if there is a biconcave function $\eta:\{(x, y) \in \mathbf{X} \times \mathbf{X}:|x-y| \leqslant$ $2\} \rightarrow \mathbf{R}$ such that $\eta(0,0)=2$ and

$$
\eta(x, y) \geqslant|x+y|
$$

If the condition $\eta(0,0)=2$ is eliminated, then the existence of such a function $\eta$ characterises the class UMD (Banach spaces with the unconditionality property for martingale differences).

\section{INTRODUCTION}

The aim of this paper is to introduce a notion that is dual to the notion of $\zeta$ convexity and to give new characterisations of UMD and Hilbert spaces with its use.

Suppose that $\mathbf{X}$ is a real or complex Banach space with norm $|\cdot|$. Recall that $\mathbf{X}$ is $\zeta$-convex if there is a biconvex function $\zeta: \mathbf{X} \times \mathbf{X} \rightarrow \mathbf{R}$ such that $\zeta(0,0)>0$ and

$$
\zeta(x, y) \leqslant|x+y| \quad \text { if } \quad|x|=|y|=1 .
$$

Biconvexity means that both $\zeta(\cdot, y)$ and $\zeta(x, \cdot)$ are convex on $\mathbf{X}$ for all $y$ and $\boldsymbol{x}$ in $\mathbf{X}$.

Burkholder, who discovered this notion, showed $[2,3,7]$ that $X$ is UMD if and only if $\mathbf{X}$ is $\zeta$-convex, and that $\mathbf{X}$ is a Hilbert space if and only if there is such a function $\zeta$ satisfying $\zeta(0,0)=1$.

\section{Received 7th April, 1992}

The author wishes to express her sincere appreciation to Professor Donald L. Burkholder for his hind guidance and careful review of this paper.

Copyright Clearance Centre, Inc. Serial-fee code: 0004-9729/93 \$A2.00+0.00. 
There is a dual notion. Let $T_{X}=\{(x, y) \in \mathbf{X} \times \mathbf{X}:|x-y| \leqslant 2\}$. Then $\mathbf{X}$ is called $\eta$-concave if there is a biconcave function $\eta: T_{X} \rightarrow \mathbf{R}$ satisfying

$$
\eta(x, y) \geqslant|x+y|
$$

Biconcavity means here that $\eta(\cdot, y)$ is concave on the closed ball with center $y$ and radius 2 with a similar condition on $\eta(x, \cdot)$. We shall show that $\mathbf{X}$ is UMD if and only if $\mathbf{X}$ is $\eta$-concave, and that $\mathbf{X}$ is a Hilbert space if and only if there is such a function $\eta$ satisfying $\eta(0,0)=2$.

\section{Some characterisations of Hilbert spaces}

If $\eta: T_{X} \rightarrow \mathbf{R}$ is a biconcave function satisfying (1.2), then

$$
\eta(0,0) \geqslant 2 \text {. }
$$

To prove this, take $x$ in $X$ with $|x|=2$ and use the concavity of $\eta(\cdot, 0)$ and (1.2) to obtain

$$
\eta(0,0) \geqslant \frac{1}{2}\{\eta(x, 0)+\eta(-x, 0)\} \geqslant \frac{1}{2}\{|x|+|-x|\}=2 .
$$

If there is a biconcave function $\eta: T_{X} \rightarrow \mathbf{R}$ satisfying (1.2), the least such function will be denoted by $\eta_{X}$ and called the optimal $\eta$-function.

Burkholder [4, p.683] found the optimal $\eta$-function for $R$. It is given by the symmetry property

$$
\eta_{R}(x, y)=\eta_{R}(y, x)=\eta_{R}(-x,-y)
$$

and

$$
\eta_{R}(x, y)= \begin{cases}x+y+(y-x+2) \mathrm{e}^{-y} & \text { if } 0 \leqslant y \leqslant x \leqslant y+2, \\ 2(1+y)-(y-x+2) \log (1+y) & \text { if }-1<y \leqslant 0, \quad-y \leqslant x \leqslant y+2 .\end{cases}
$$

Note that $\eta_{R}(0,0)=2$.

If $H$ is a Hilbert space with higher linear dimension over the real field $\mathbf{R}$, then the optimal $\eta$-function has an entirely different formula:

THEOREM 1. Suppose that $\mathbf{H}$ is a real or complex Hilbert space with the linear dimension over the real field $\mathbf{R}$ greater than or equal to two. Then the optimal $\eta$ function is given by

$$
\eta_{H}(x, y)=2 \sqrt{1+(x, y)}
$$


where $(x, y)$ denotes the real part of the inner product of $x$ and $y$.

Proof: Let $u(x, y)=2 \sqrt{1+(x, y)}$. Pick $(x, y)$ in $T_{H}$. Then $|x-y| \leqslant 2$ and

$$
\begin{aligned}
1+(x, y) & =1+(x, x-(x-y)) \\
& =|x|^{2}-(x, x-y)+1 \\
& \geqslant|x|^{2}-2|x|+1 \\
& =(|x|-1)^{2} \geqslant 0 .
\end{aligned}
$$

Since the mapping $(x, y) \mapsto 1+(x, y)$ is biaffine and nonnegative on $\boldsymbol{T}_{H}$, and the mapping $t \mapsto \sqrt{t}$ is concave on $[0, \infty)$, the function $u$ is biconcave on $T_{H}$.

It is easy to see that $u$ satisfies (1.2):

$$
\begin{aligned}
\{2 \sqrt{1+(x, y)}\}^{2} & =4+4(x, x-(x-y)) \\
& =4|x|^{2}-4(x, x-y)+4 \\
& \geqslant 4|x|^{2}-4(x, x-y)+|x-y|^{2} \\
& =|x+y|^{2} .
\end{aligned}
$$

Note that equality holds in the above inequality if $|x-y|=2$.

What remains to be shown is that $u$ is optimal. If $|x-y|=2$, then $u(x, y)=$ $|x+y|$ as shown above. So $u(x, y) \leqslant \eta_{H}(x, y)$ on the boundary of $T_{H}$. Suppose that $|x-y|<2$. Since the dimension of $H$ is greater than or equal to two, there is a point $z$ in the unit sphere $S_{H}$ of $\mathrm{H}$ which is orthogonal to $x$.

The function $v$ defined on $\mathrm{R}$ by $v(s)=|x-y-s z|$ is continuous, $v(0)=|x-y|<$ 2 , and $v(s) \geqslant 2$ for $|s|$ large. So there are two real numbers $s_{1}$ and $s_{2}$ so that $s_{1}<0<s_{2}$ and $\left|x-y-s_{i} z\right|=2$ for $i=1,2$. Since $x$ is orthogonal to $z$, the mapping $s \mapsto u(x, y+s z)$ is constant on the closed interval $\left[s_{1}, s_{2}\right]$. Therefore

$$
u(x, y)=u\left(x, y+s_{i} z\right) \leqslant \eta_{H}\left(x, y+s_{i} z\right) \quad \text { for } \quad i=1,2 .
$$

Let $\alpha=s_{2} /\left(s_{2}-s_{1}\right)$. Then $\alpha s_{1}+(1-\alpha) s_{2}=0$ and

$$
\begin{aligned}
u(x, y) & =\alpha u(x, y)+(1-\alpha) u(x, y) \\
& =\alpha u\left(x, y+s_{1} z\right)+(1-\alpha) u\left(x, y+s_{2} z\right) \\
& \leqslant \alpha \eta_{H}\left(x, y+s_{1} z\right)+(1-\alpha) \eta_{H}\left(x, y+s_{2} z\right) \\
& \leqslant \eta_{H}\left(x, \alpha\left(y+s_{1} z\right)+(1-\alpha)\left(y+s_{2} z\right)\right) \\
& =\eta_{H}(x, y)
\end{aligned}
$$


where we have used the orthogonality of $x$ and $z$ and the concavity of $\eta_{H}(x, \cdot)$. Therefore $u(x, y) \leqslant \eta_{H}(x, y)$ for all $(x, y) \in T_{X}$. This completes the proof of Theorem 1.

Hilbert spaces are therefore $\eta$-concave. Moreover if $\mathbf{X}$ is a Hilbert space, there is a function attaining the lower bound 2 in (2.1) regardless of the dimension of $\mathbf{X}$. A natural question is: Does this property characterise Hilbert space?

Before we answer this question, let us consider the following problem: Let $x$ be a point in the Banach space $\mathrm{X}$ and $Y$ an $\mathrm{X}$-valued Bochner integrable function on the Lebesgue unit interval. Denote by $E Y$ the integral of $Y$ on $[0,1)$. (See [10] for background information on the Bochner integral.) Assume that $E Y=0$ and $|x-Y| \leqslant 2$ almost everywhere. Then how large can $E|x+Y|$ be?

LEMma 1. Suppose that $\mathrm{X}$ is an $\eta$-concave Banach space. If $x \in \mathrm{X}$ and $Y$ is an $\mathrm{X}$-valued Bochner integrable function on the Lebesgue unit interval satisfying $E Y=0$ and the inequality $|x-Y| \leqslant 2$ almost everywhere, then

$$
E|x+Y| \leqslant \eta(0,0)
$$

for all biconcave functions $\eta: T_{X} \rightarrow \mathbf{R}$ satisfying (1.2).

Proof: Take $x$ and $Y$ as above and let $\eta$ be a biconcave function on $T_{X}$ satisfying (1.2). We can assume, without loss of generality, that $|x-Y| \leqslant 2$ everywhere. Note that

$$
|x|=|E(x-Y)| \leqslant E|x-Y| \leqslant 2 .
$$

By (1.2) and Jensen's inequality applied to the continuous and concave function $\eta(x, \cdot)$,

$$
E|x+Y| \leqslant E \eta(x, Y) \leqslant \eta(x, E Y)=\eta(x, 0)
$$

where the continuity comes from the fact that $\eta$ is bounded from below. Replacing $\eta$ by the mapping $(x, y) \mapsto \eta(x, y) \wedge \eta(-x,-y)$, if necessary, we can assume that

$$
\eta(x, y)=\eta(-x,-y)
$$

By (2.4) and concavity of $\eta(\cdot, 0)$,

$$
\eta(x, 0)=\frac{1}{2}\{\eta(x, 0)+\eta(-x, 0)\} \leqslant \eta(0,0),
$$

which completes the proof of Lemma 1.

In particular, if $\mathrm{X}$ is a Hilbert space, then Theorem 1 and the function $\eta_{R}$ defined by symmetry and (2.2) gives $E|x+Y| \leqslant 2$. A natural question is: Does $E|x+Y| \leqslant 2$ characterise Hilbert space? 
TheOREM 2. Suppose that $\mathrm{X}$ is a real or complex Banach space. If

$$
E|x+Y| \leqslant 2
$$

for all $x$ in $\mathrm{X}$ and all $\mathrm{X}$-valued Bochner integrable functions $Y$ on the Lebesgue unit interval satisfying $E Y=0$ and $|x-Y| \leqslant 2$ almost everywhere, then $X$ is a Hilbert space.

Proof: Suppose that $X$ is not a Hilbert space. We shall find $x$ and $Y$ so that $E Y=0,|x-Y| \leqslant 2$ everywhere, but $E|x+Y|>2$. To prove this, we can assume that $\mathrm{X}$ is a Banach space over the real field. We need the following two lemmas from the theory of convex bodies; see [11] and [1] for the proofs.

Lemma 2. Suppose that $X$ is a two-dimensional real Banach space. Then the norm of $\mathrm{X}$ is generated by an inner product if and only if the unit sphere of $\mathrm{X}$ is an ellipse.

LEMMA 3. If $C$ is a symmetric (about the origin) closed convex curve in the plane, then there exists a unique ellipse of minimal area circumscribed about $C$. The minimal circumscribed ellipse touches $C$ in at least four points that are symmetric pairwise.

Without loss of generality, we can assume that the dimension of $\mathbf{X}$ is equal to two. Let $S_{X}$ denote the unit sphere of $\mathrm{X}$. Then, by Lemma 3 , there is a unique ellipse $S_{0}$ of minimal area circumscribed about $S_{X}$ with at least four contact points that are symmetric pairwise. After some affine transformations, we can assume that $S_{0}$ is the unit circle. Denote by $\|\cdot\|$ the norm induced by $S_{0}$. Let $\pm A$ and $\pm C$ denote four contact points with no contact points in the interior of the arc $\overparen{A C}$. The existence of such points is assured by Lemmas 2 and 3. Let $\theta=1 / 2 \angle A O C$, one half of the angle determined by the line segments $\overline{O A}$ and $\overline{O C}$. Here $O$ denotes the origin of $\mathrm{X}$. We can assume that $0<2 \theta \leqslant \pi / 2, A=(1,0)$, and $C=(\cos 2 \theta, \sin 2 \theta)$. Let $D=s(\cos \theta, \sin \theta)$ where $s$ is a positive number satisfying $|s(\cos \theta, \sin \theta)|=1$. Accordingly $s<1$.

Define $x$ and $Y$ on $(-s, s)$ by

$$
x(t)=t(\cos \theta, \sin \theta)
$$

and

$$
\begin{aligned}
Y(t)= & (2+t \cos \theta, t \sin \theta) I_{[0, p)}+(2 \cos 2 \theta+t \cos \theta, 2 \sin 2 \theta+t \sin \theta) I_{[p, 2 p)} \\
& -(2 s-t)(\cos \theta, \sin \theta) I_{[2 p, 1)}
\end{aligned}
$$

where $p=(2 s-t) /(4(\cos \theta+s))$ and $I_{[a, b)}$ denotes the indicator function of the interval $[a, b)$. Then, for any real number $t \in(-s, s)$, we have $E Y(t)=0,|Y-x|=2$ everywhere, and

$$
\begin{aligned}
Y(t)+x(t) & =2(1+t \cos \theta, t \sin \theta) I_{[0, p)}+2(\cos 2 \theta+t \cos \theta, \sin 2 \theta+t \sin \theta) I_{[p, 2 p)} \\
& -2(s-t)(\cos \theta, \sin \theta) I_{[2 p, 1)} .
\end{aligned}
$$


Let $f$ and $g$ be functions defined on $(-s, s)$ by

$$
\begin{aligned}
f(t) & =E|Y(t)+x(t)| \\
& =2 p|(1+t \cos \theta, t \sin \theta)|+2 p|(\cos 2 \theta+t \cos \theta, \sin 2 \theta+t \sin \theta)|+2(1-2 p) \frac{s-t}{s}, \\
g(t) & =2 p\|(1+t \cos \theta, t \sin \theta)\|+2 p\|(\cos 2 \theta+t \cos \theta, \sin 2 \theta+t \sin \theta)\|+2(1-2 p) \frac{s-t}{s} .
\end{aligned}
$$

Then

$$
\begin{aligned}
& f(t) \geqslant g(t) \quad \text { with } f(0)=g(0)=2, \\
& g(t)=\frac{2 s-t}{\cos \theta+s}\left(1+2 t \cos \theta+t^{2}\right)^{\frac{1}{2}}+\frac{2 \cos \theta+t}{\cos \theta+s} \cdot \frac{s-t}{s},
\end{aligned}
$$

and $\quad g^{\prime}(0)=\frac{2 \cos \theta}{s(\cos \theta+s)}\left(s^{2}-1\right)<0$ since $0<s<1$ and $0<\theta \leqslant \pi / 4$.

So there is a number $\epsilon \in(0,2]$ such that $f(t) \geqslant g(t)>g(0)$ if $-\epsilon<t<0$. Let $x=x(t)$ and $Y=Y(t)$ for a real number $t \in(-\epsilon, 0)$. Then $x$ is a point in $X$ and $Y$ is an $\mathrm{X}$-valued simple function satisfying $E Y=0,|x-Y|=2$ everywhere, and $E|x+Y|>2$. This completes the proof of Theorem 2.

An immediate consequence of Theorem 2 is the following biconcave-function characterisation of Hilbert spaces:

THEOREM 3. Suppose that $\mathrm{X}$ is a real or complex Banach space. If there is a biconcave function $\eta: T_{X} \rightarrow \mathbf{R}$ such that $\eta(0,0)=2$ and (1.2) is satisfied, then $\mathbf{X}$ is a Hilbert space.

Proof: Suppose on the contrary that $X$ is not a Hilbert space. Let $\eta: T_{X} \rightarrow \mathbf{R}$ be a biconcave function satisfying (1.2). As before we can assume that $\eta$ satisfies (2.4).

By Theorem 2 and its proof, there exist a point $x \in \mathrm{X}$ and an $\mathrm{X}$-valued simple function $Y$ so that $E Y=0,|x-Y|=2$ everywhere, and $E|x+Y|>2$. Therefore by (2.3)

$$
\eta(0,0) \geqslant E|x+Y|>2 .
$$

This completes the proof of Theorem 3.

\section{A CHARACTERISATION OF THE CLASS UMD}

It is well known that the UMD spaces provide the right setting for many problems in analysis. See the work of Burkholder, McConnell, and Bourgain in the early 1980s and the later work of many others referenced in [7] and [8]. We shall recall the definition, which will be needed later, of the UMD property. 
Let $\left(\Omega, F_{\infty}, P\right)$ be a probability space. In fact, without loss of generality, let $\Omega=[0,1)$ and $P$ be Lebesgue measure on the Borel $\sigma$-field $F_{\infty}$ of $\Omega$. Let $F=$ $\left(F_{n}\right)_{n \geqslant 0}$ be a nondecreasing sequence of sub- $\sigma$-fields of $F_{\infty}$ and $f=\left(f_{n}\right)_{n \geqslant 0}$ be an $\mathrm{X}$-valued martingale adapted to $F$ with difference sequence $d=\left(d_{n}\right)_{n \geqslant 0}: f_{n}=\sum_{k=0}^{n} d_{k}$ where $d_{k}: \Omega \rightarrow \mathrm{X}$ is Bochner integrable and measurable with respect to $F_{k}$ with $E\left(d_{k} \mid F_{k-1}\right)=0$ almost everywhere. If $f$ converges almost everywhere, let $f_{\infty}$ denote its almost everywhere limit. The maximal function $f^{*}$ of $f$ is defined by $f^{*}(s)=$ $\sup _{n}\left|f_{n}(s)\right|, s \in[0,1)$, and the $L^{p}$-norm of $f$ by $\|f\|_{p}=\sup _{n}\left(E\left|f_{n}\right|^{p}\right)^{1 / p}, 1 \leqslant p<\infty$, and $\|f\|_{\infty}=\sup _{n \geqslant 0}\left\|f_{n}\right\|_{\infty}$.

A real or complex Banach space $\mathbf{X}$ has the unconditionality property for martingale differences, in short, $\mathbf{X}$ is $\mathrm{UMD}$, if all $\mathbf{X}$-valued martingale difference sequences are unconditional in the Lebesgue-Bochner space $L^{p}([0,1), X)$ for $1<p<\infty$ : There exists a positive constant $\beta$ so that if $\boldsymbol{d}$ is an $\mathrm{X}$-valued martingale difference sequence, $\epsilon$ is a sequence with values in $\{1,-1\}$, and $n$ is a nonnegative integer, then

$$
\left\|\sum_{k=0}^{n} \epsilon_{k} d_{k}\right\|_{p} \leqslant \beta\left\|\sum_{k=0}^{n} d_{k}\right\|_{p} .
$$

Here $g=\left(g_{n}\right)_{n \geqslant 0}$, where $g_{n}=\sum_{k=0}^{n} \epsilon_{k} d_{k}$, is called the transform of the martingale $f$ by the sequence $\epsilon$. The constant $\beta$ depends only on $p$ and $X \operatorname{not}$ on $d, \epsilon$, or $n$. The least such $\beta$, denoted by $\beta_{p}(\mathbf{X})$, is called the UMD constant of $\mathbf{X}$.

THEOREM 4. A Banach space $\mathrm{X}$ is $U M D$ if and only if it is $\eta$-concave.

Let $Z=\left(Z_{n}\right)_{n \geqslant 0}$ be a martingale with values in $\mathrm{X} \times \mathrm{X}$. Write $Z_{n}=\left(X_{n}, Y_{n}\right)$ where both $X_{n}$ and $Y_{n}$ have their values in $\mathrm{X}$. Then $Z$ is a zigzag martingale if, for every positive integer $n$, either

$$
X_{n}-X_{n-1} \equiv 0 \text { or } Y_{n}-Y_{n-1} \equiv 0
$$

The martingale $Z$ is simple if each term $Z_{n}$ is a simple function and $Z_{n}=Z_{n+1}=$ $\cdots=Z_{\infty}$ for some nonnegative integer $n$.

The notion of $\eta$-concavity arises in connection with an upper solution to a nonclassical boundary value problem posed by Burkholder (see [4] for the real case and [7] for the Banach space case). If $\mathbf{X}$ is $\eta$-concave then the least biconcave function on $T_{X}$ satisfying (1.2) can be represented in terms of zigzag martingales as follows.

For $(x, y) \in T_{X}$, let $\mathcal{Z}(x, y)$ denote the set of all simple zigzag martingales $Z$, defined on the Lebesgue unit interval, such that $Z_{0}=(x, y)$ and $Z_{n}=\left(X_{n}, Y_{n}\right)$ has all 
its values in $T_{X}$. Then it is easy to see that $\mathcal{Z}(x, y)$ is nonempty for all $(x, y) \in T_{X}$. Let

$$
U(x, y)=\sup \left\{E\left|X_{\infty}+Y_{\infty}\right|: Z \in \mathcal{Z}(x, y)\right\} .
$$

Lemma 4. Suppose that the Banach space $\mathrm{X}$ is $\eta$-concave. Then the function $U$ is the optimal $\eta$-function $\eta_{X}$.

See [7] for the proof and for the more general setting. The relevance of upper solutions and lower solutions to nonclassical boundary value problems of this type is revealed in $[4,5,6]$, and $[7]$. The relationship between $\zeta$-convexity and a lower solution is described in Chapter 5 of [7].

From Lemma 4 it follows that $\mathrm{X}$ is $\eta$-concave if and only if $U(x, y)<\infty$ for all $(x, y) \in T_{X}$. Furthermore $\eta$-concavity is assured by the finiteness of the value of $U(0,0)$ :

Lemma 5. A Banach space $\mathrm{X}$ is $\eta$-concave if and only if $U(0,0)$ is finite.

From time to time, it is more convenient to work with the following representation:

$$
U(0,0)=2 \sup \left\{\|f\|_{1}:(f, g) \in \mathcal{M}(0,0)\right\} .
$$

This comes from the one-to-one correspondence between the set $\mathcal{Z}(0,0)$ and the set $\mathcal{M}(0,0)$, where the set $\mathcal{M}(0,0)$ consists of all pairs $(f, g)$ of $X$-valued simple martingales defined on the Lebesgue unit interval starting at 0 with $g$ a transform of $f$ by a sequence $\epsilon$ of numbers in $\{1,-1\}$ with the property that $g$ is uniformly bounded in norm by 1 ; see [4].

Finally we need the following lemma from [3]:

LEMMA 6. Suppose that

$$
\|f\|_{\infty}<\infty \text { implies that } P\left(g^{*}<\infty\right)>0
$$

for all $f$ and $g$ where $g$ is the transform of an X-valued martingale $f$ by a sequence of numbers in $\{1,-1\}$. Then $\mathrm{X}$ is UMD.

Proof of Theorem 4: Suppose that $\mathbf{X}$ is UMD. Select $(f, g) \in \mathcal{M}(0,0): f$ is a martingale on the Lebesgue unit interval with $f_{0} \equiv 0$, and $g$ is a transform of $f$ by a sequence of numbers in $\{1,-1\}$ with the property that $g$ is uniformly bounded in norm by 1 . Then

$$
\|f\|_{1} \leqslant\|f\|_{2} \leqslant \beta_{2}(\mathrm{X})\|g\|_{2} \leqslant \beta_{2}(\mathrm{X})\|g\|_{\infty} \leqslant \beta_{2}(\mathrm{X}) .
$$

Here $\beta_{2}(X)$, the UMD constant of $\mathbf{X}$, is finite because $\mathbf{X}$ is UMD. The first and the third inequalities hold because the mapping $p \mapsto\|f\|_{p}$ is nondecreasing on $(0, \infty]$ 
for any Bochner integrable function $f$ defined on $[0,1)$. It follows from (3.1) that $U(0,0) \leqslant 2 \beta_{2}(X)$. By Lemma 5 , the Banach space $\mathrm{X}$ is $\eta$-concave.

Suppose that $\mathbf{X}$ is $\eta$-concave. Let $f$ be an $\mathbf{X}$-valued martingale with $\|f\|_{\infty}<\infty$. We can assume that $\|f\|_{\infty} \leqslant 1$. Let $g$ be a transform of $f$ by a sequence $\epsilon$ of numbers in $\{1,-1\}$. Since $f$ is a martingale transform of $g$ by the same sequence $\epsilon$, it follows from (3.1) and Lemma 5 that $2\|g\|_{1} \leqslant U(0,0)<\infty$. By Doob's weak-type inequality [9] applied to a nonnegative submartingale $\left(\left|g_{n}\right|\right)_{n \geqslant 0}$,

$$
P\left(g^{*} \geqslant \lambda\right) \leqslant \frac{1}{\lambda}\|g\|_{1}, \quad \lambda>0 .
$$

Letting $\lambda \rightarrow \infty$, we obtain $P\left(g^{*}<\infty\right)=1$. By Lemma 6 , the Banach space $\mathbf{X}$ is UMD, which completes the proof of Theorem 1 .

\section{REFERENCES}

[1] D. Amir, Characterizations of inner product spaces, Operator theory: Advances and applications 20 (Birkhäuser Verlag, Boston, 1986).

[2] D.L. Burkholder, 'A geometrical characterization of Banach spaces in which martingale difference sequences are unconditional', Ann. Probab. 9 (1981), 997-1011.

[3] D.L. Burkholder, 'Martingale transforms and the geometry of Banach spaces', in Proceedings of the Third International Conference on Probability in Banach Spaces, Tufts University, 1980, Lecture Notes in Mathematics 860 (Springer-Verlag, Berlin, Heidelberg, New York, 1981), pp. 35-50.

[4] D.L. Burkholder, 'Boundary value problems and sharp inequalities for martingale transforms', Ann. Probab. 12 (1984), 647-702.

[5] D. L. Burkholder, 'An elementary proof of an inequality of R.E.A.C. Paley', Bull. London Math. Soc. 17 (1985), 474-478.

[6] D.L. Burkholder, 'An extension of a classical martingale inequality', in Probability Theory and Harmonic Analysis, (J.A. Chao and W.A. Woyczynski, Editors) (Marcel Dekker, New York (1986)), pp. 21-30.

[7] D.L. Burkholder, 'Martingales and Fourier analysis in Banach spaces', in C.I.M.E. Lectures, Varenna (Como), Italy, 1985, Lecture Notes in Math. 1206 (Springer-Verlag, Berlin, Heidelberg, New York, 1986), pp. 61-108.

[8] D.L. Burkholder, 'Explorations in Martingale theory and its applications', in Saint-Flour Lectures, 1989, Lecture Notes in Math. 1464 (Springer-Verlag, Berlin, Heidelberg, New York, 1991), pp. 1-66.

[9] J.L. Doob, Stochastic processes (Wiley, New York, 1953).

[10] J. Diestel and J.J. Uhl, Vector measures, Math. Surveys 15 (Amer. Math. Soc., Providence, Rhode Island, 1977).

[11] V.I. Istrăł̧escu, Inner product structures (D. Reidel Publishing Company, Boston, 1987). 
Department of Mathematics

Sungwha University

381-7 Samyong-Dong Chonahn

330-150 Republic of Korea 